\title{
Low-Flow Domiciliary Oxygen as a Mechanism of Ongoing Oxidative Stress
}

\author{
Jill M Stulce, Clarence Biddle, and Charles Vacchiano
}

\begin{abstract}
BACKGROUND: Oxidative stress occurs when imbalances exist between the production of oxygen free radicals and endogenous antioxidants that neutralize their harmful effects, causing irreversible tissue damage. Oxygen free radicals readily interact with DNA, proteins, and lipids, instigating conformational changes to cellular structures and leading to derangement and dysfunction. Oxidative stress is a key feature in the pathology of COPD. As disease progression occurs, supplemental oxygen is often warranted to ameliorate dyspnea. It has been established that supplemental oxygen at $>0.60 \mathrm{~F}_{\mathrm{IO}}$ is an instigator of oxidative stress. We sought to determine whether chronic exposure to low-flow domiciliary oxygen served as a mechanism of ongoing oxidative stress in this patient population. This study serves to inform best practices for low-flow domiciliary oxygen therapy. METHODS: We utilized prospective data collection for this study. The exhaled-breath condensate (EBC) of 52 subjects with $\mathrm{COPD}\left(\mathrm{FEV}_{1}<70 \%\right.$ of predicted normal for age and gender, height, weight, and smoking history) was evaluated for isofuran (IsoF), an oxidative stress biomarker synthesized in response to elevated tissue oxygen tension. The active control group $(n=26)$ was compared to the active treatment group receiving low-flow domiciliary oxygen for $\geq 6 \mathrm{~h} / \mathrm{d}(n=26)$. RESULTS: The active control group generated a mean \pm SD EBC IsoF level of $35.81 \pm 25.0 \mathrm{pg} / \mathrm{mL}$ compared to the active treatment group's level of $51.37 \pm 42.2 \mathrm{pg} / \mathrm{mL}(P=.057)$. CONCLUSIONS: In subjects diagnosed with advanced COPD, chronic exposure to supplemental oxygen therapy at concentrations $\leq 36 \%$ did not appear to induce oxidative stress based on EBC IsoF levels. Our findings do not substantiate that chronic exposure to supplemental oxygen at concentrations $<36 \%$ instigates oxidative stress, and, therefore, changes to current domiciliary oxygen therapy prescription practices are not warranted. Key words: oxidative stress; reactive oxygen species; antioxidants; lipid peroxidation; exhaled-breath condensate; isofuran; low-flow domiciliary oxygen. [Respir Care 2019;64(11):1387-1391. @ 2019 Daedalus Enterprises]
\end{abstract}

\section{Introduction}

It is well understood that oxidative stress is central to the pathogenesis of COPD. ${ }^{1}$ Oxidative stress reflects an imbalance between the systemic production of reactive oxygen species and the body's ability, through antioxidant systems, to neutralize the reactive intermediates or

\footnotetext{
Dr Stulce is affiliated with the College of Arts and Sciences, Department of Nurse Anesthesia, Webster University, St. Louis Missouri. Dr Biddle is affiliated with the Department of Nurse Anesthesia, Virginia Commonwealth University, Richmond Virginia. Dr Vacchiano is affiliated with the School of Nursing, Nurse Anesthesia Program, Duke University, Durham North Carolina.
}

The authors have disclosed a relationship with the AANA Foundation. to repair the resulting damage. Reactive oxygen species are produced by a variety of endogenous biochemical processes and are highly bioreactive, and lipids, proteins, and DNA are vulnerable targets. ${ }^{2}$ The production of reactive oxygen species can also be stimulated by exogenous sources such as environmental pollutants, radiation exposure, and hyperoxia. ${ }^{3}$ The antioxidant capacity in individuals with COPD is considerably reduced over the course of their disease due to smoking

\footnotetext{
Correspondence: Jill M Stulce PhD CRNA, Webster University, 8274 Big Bend Blvd, Office 105, St. Louis, MO, 63119. E-mail: jillstulce02@webster.edu.
}

DOI: $10.4187 /$ respcare. 05618 
and exacerbations of the disease, making this population subset particularly vulnerable to the widespread damage induced by oxidative stress. ${ }^{4}$

Oxygen is central to all human metabolic processes, but as a drug it has a maximum physiologic benefit before toxicity ensues in a time-dependent manner. Supplemental oxygen therapy can result in hyperoxia and oxygen toxicity. ${ }^{2,5}$ Oxygen toxicity occurs when $\mathrm{P}_{\mathrm{aO}_{2}}$ exceeds what is breathed under normal atmospheric conditions. Tissues exposed to a persistent state of supraphysiologic concentrations of oxygen (ie, hyperoxia) will produce reactive oxygen species, making the host susceptible to the deleterious effects of oxidative stress. Length of exposure, atmospheric pressure, and $\mathrm{F}_{\mathrm{IO}_{2}}$ determine the cumulative oxidant load that results in toxicity. ${ }^{2}$

Isoprostanes (IsoPs) are prostaglandin-like compounds formed in vivo from the free radical-catalyzed peroxidation of essential fatty acids (primarily arachidonic acid) without the direct action of cyclooxygenase enzymes. ${ }^{6}$ Since their discovery, IsoPs have been used in numerous studies and are considered accurate and reliable biomarkers of lipid peroxidation in human models of oxidative stress. ${ }^{7}$ The process of oxidative stress produces many metabolic byproducts that provide a variety of biomarkers, such as hydrocarbons, hydrogen peroxide, alkanes (ethane and pentane), and IsoPs, but their utility is limited by sensitivity and specificity issues. In the case of IsoPs, their production essentially halts in the presence of supraphysiologic oxygen tissue tensions. ${ }^{8}$ In 2002, a biosynthetic relative of the IsoPs, isofurans (IsoFs), was isolated. It was determined that IsoF formation is favored during periods of increased oxygen tissue tensions. ${ }^{8}$ The identification of a biomarker specific to changes in oxygen tissues tensions affords researchers a unique opportunity to further evaluate the role of oxygen therapy as an instigator of oxidative stress.

Low-flow domiciliary oxygen is a well-established and widely prescribed therapy, with clear evidence of benefits such as prolonging life and ameliorating respiratory distress. ${ }^{9,10}$ The exact concentration of oxygen that is toxic to the lungs is unknown and is likely affected by a number of variables, such as age, nutrition, comorbidities, and environmental exposures. ${ }^{11}$ It has been generally accepted, however, that an $\mathrm{F}_{\mathrm{IO}_{2}}<0.40$ at sea level does not discernably contribute to oxidative pathology. ${ }^{12,13}$ Two studies have challenged this convention by demonstrating significant increases in exhaled-breath oxidative stress biomarkers (eg, breath-methylated alkane contour, 8-IsoP, IL-6) following brief exposure to $28 \% \mathrm{~F}_{\mathrm{IO}_{2}}$ for $30 \mathrm{~min}$ and $60 \mathrm{~min}$, respectively. ${ }^{14,15}$ It was postulated for this study that the unique quality of IsoF production related to supraphysiologic oxygen conditions could provide clarity to this conundrum.

\section{QUICK LOOK}

\section{Current knowledge}

Short exposure (6-8 h) to high concentrations (ie, $>40 \%$ ) of oxygen can result in oxidative stress-induced lung injury. Similar injury has not been reported in patients receiving long-term domiciliary oxygen therapy.

\section{What this paper contributes to our knowledge}

The results of this study suggest that risk of oxidative stress is low in subjects with COPD exposed to low concentrations of domiciliary oxygen. Thus, no changes in prescription are warranted in this regard.

\section{Methods}

The purpose of this study was to identify the potential risk of low-flow domiciliary oxygen therapy as an instigator of oxidative stress in a population subset already subject to oxidative pathology as evidenced by the presence of IsoF in exhaled breath condensate (EBC). Data collection occurred at Phelps County Regional Medical Center in Rolla, Missouri. A 1-tailed $t$ test analysis using GraphPad Prism 6.0 was used to compare group differences between subjects diagnosed with advanced COPD $\left(\mathrm{FEV}_{1} / \mathrm{FVC}<0.70\right)$ not receiving home oxygen therapy and subjects with advanced COPD who do receive home oxygen therapy. A 1-tailed $t$ test was used because only an increase in the observed biomarker was expected. A $\mathrm{G}^{*}$ Power analysis $(\alpha=0.05, \beta=0.80$, effect size 0.8 ; UCLA Institute for Digital Research and Education, Los Angeles, California) indicated that a sample size of 52 subjects was needed to sufficiently power the study with the assumption that IsoF production mirrors IsoP production in normoxic environments and exceeds in production during hyperoxic environments. In the statistical analysis, $P \leq .05$ was accepted for significance. After institutional review board approval from Phelps County Regional Medical Center, groups of individuals diagnosed with advanced COPD were closely matched with regard to age, gender, height, weight, ethnicity, and the spirometric results of $\mathrm{FEV}_{1}, \mathrm{FVC}, \mathrm{FEV}_{1} / \mathrm{FVC}$, and forced expiratory flow during the middle half of the FVC maneuver $\left(\mathrm{FEF}_{25-75 \%}\right)$. The resulting enrollment included 26 participants in the active control group, who received no supplemental oxygen therapy, and 26 participants in the active treatment group, who received supplemental oxygen for $\geq 6 \mathrm{~h} / \mathrm{d}$ (Table 1).

The EBC was collected from each study participant using the RTube collection device (Respiratory Research, Charlottesville, Virginia). Each sample was immediately 
Table 1. Demographic Data

\begin{tabular}{lccc}
\hline \hline & $\begin{array}{c}\text { Control Group } \\
\text { (COPD Without } \\
\text { Oxygen) }\end{array}$ & $\begin{array}{c}\text { Treatment Group } \\
\text { (COPD With } \\
\text { Oxygen) }\end{array}$ & $P$ \\
\hline Age, y & $62 \pm 29$ & $65 \pm 16.5$ & .37 \\
Female, $n$ & 11 & 16 & .17 \\
Height, cm & $167.6 \pm 12.7$ & $165.1 \pm 19.1$ & .31 \\
Weight, kg & $86.2 \pm 39.7$ & $98.4 \pm 54.0$ & .09 \\
Caucasian, $n$ & 26 & 26 & \\
Smoking history, $n(\%)$ & $22(84.6)$ & $22(84.6)$ & \\
Current smokers, $n(\%)$ & $13(50.0)$ & $0(0)$ & \\
& & & \\
$n=26$ subjects in each group. Age, height, and weight are presented as mean \pm SD. & \\
\hline
\end{tabular}

Table 2. Pulmonary Function Spirometry Results

\begin{tabular}{lccc}
\hline \hline & $\begin{array}{c}\text { Control Group } \\
\text { (COPD Without Oxygen) }\end{array}$ & $\begin{array}{c}\text { Treatment Group } \\
\text { (COPD With Oxygen) }\end{array}$ & $P$ \\
\hline $\mathrm{FEV}_{1}$ & $60.19 \pm 13.92$ & $60.08 \pm 19.99$ & .98 \\
$\mathrm{FVC}$ & $75.81 \pm 18.71$ & $70.42 \pm 23.46$ & .36 \\
$\mathrm{FEV}_{1} / \mathrm{FVC}$ & $64.63 \pm 14.58$ & $68.38 \pm 14.33$ & .35 \\
$\mathrm{FEF}_{25-75 \%}$ & $34.93 \pm 20.35$ & $51.38 \pm 30.90$ & .03
\end{tabular}

Values are presented as mean $\pm \mathrm{SD}$.

$\mathrm{FEF}_{25-75 \%}=$ forced expiratory flow during the middle half of the $\mathrm{FVC}$ maneuver

stored on dry ice and maintained at a temperature $-80^{\circ} \mathrm{C}$ until analysis was performed in the Vanderbilt University Core Eicosanoid Labs. Samples were analyzed using gas chromatography and mass spectrometry to accurately identify and quantify EBC IsoF. Results were reported in $\mathrm{pg} / \mathrm{mL}$.

\section{Results}

No significant differences were detected between groups with regard to age $(P=.37)$, gender $(P=.17)$, height $(P=.31)$, weight $(P=.09)$, ethnicity (all were Caucasian), smoking history (22 of 26 subjects in each group), or pulmonary function spirometry results of $\mathrm{FEV}_{1}(P=.98)$, FVC $(P=.36)$, and $\mathrm{FEV}_{1} / \mathrm{FVC}(P=.35)$; the exception was $\operatorname{FEF}_{25-75 \%}(P=.03)$ (Table 2). A 1-tailed $t$ test was performed to determine the mean difference in $\mathrm{EBC}$ IsoF levels between the active control and active treatment groups. The active control group generated a mean EBC IsoF level of $35.81 \pm 25.0 \mathrm{pg} / \mathrm{mL}$ ( $\pm \mathrm{SD}$ ) compared to the active treatment group mean EBC IsoF level of $51.37 \pm 42.2$ $\mathrm{pg} / \mathrm{mL}(P=.057)$ (Fig. 1).

\section{Discussion}

Low-flow domiciliary oxygen therapy is an established treatment strategy for individuals with advanced respira-

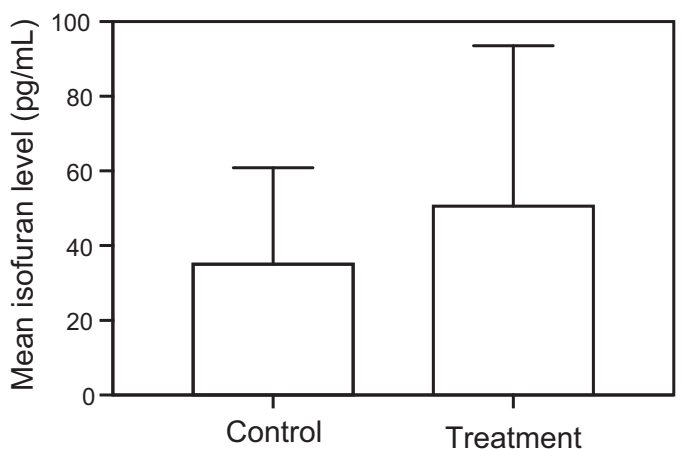

Fig. 1. Isofuran levels in the control group versus treatment group. Data are shown as mean \pm SD.

tory disease, such as severe COPD, to ameliorate the symptoms of dyspnea. At $\mathrm{F}_{\mathrm{IO}_{2}}>0.40$, supplemental oxygen is a known instigator of oxidative stress. ${ }^{12,13}$ The purpose of this study was to elucidate whether low-flow domiciliary oxygen therapy contributes to ongoing oxidative stress in the lungs of individuals with $\mathrm{COPD}$ at $\mathrm{F}_{\mathrm{IO}_{2}}<0.36$. This study did not demonstrate a significant difference between the closely matched groups with regard to low-flow domiciliary oxygen therapy. As a result of this finding, no changes to current domiciliary oxygen prescription are warranted at this time.

The risks generally reported to be associated with domiciliary oxygen therapy range from dry or bloody nose, skin irritation, fatigue, and morning headaches, to fire hazards, with the most consequential being a decrease in respiratory drive resulting in hypercapnia (https://www. nhlbi.nih.gov/health-topics/oxygen-therapy, Accessed February 6, 2019). Approximately 1 million patients receive domiciliary oxygen prescriptions through Medicaid at a cost exceeding $\$ 2$ billion annually. ${ }^{2}$ Because domiciliary oxygen therapy is costly, Medicare reimbursement for its prescription is regulated. More than ever, medical researchers are obligated to ensure that costly treatment modalities are safe and effective. Identifying mechanisms to assess prescription efficacy is warranted. No research to date has been conducted utilizing IsoF as a marker of oxidative stress in the lungs of COPD subjects; in fact, no research has been conducted utilizing any biomarker to determine if chronic domiciliary oxygen therapy presents an oxidative risk. The results of this study will also serve to provide benchmark information about baseline IsoF levels in the COPD population.

IsoF production is currently understood to occur in response to elevated oxygen tensions. ${ }^{8}$ Elevated oxygen tension levels within the body can occur from both endogenous and exogenous sources. This study aimed to discern whether oxidative stress was occurring as a result of prescribed low-flow domiciliary oxygen therapy, which is an exogenous source of oxygen. A simple, first-step approach 
to determining this potential was to create closely matched groups with regard to age, gender, COPD diagnosis, and ethnicity and to compare the mean IsoF levels between the active control group (not receiving supplemental oxygen) and treatment groups (receiving supplemental oxygen).

When considering variables that would most likely affect the development of oxidative stress within the lungs of patients with COPD receiving domiciliary oxygen, time and concentration are intuitive. However, in the current study, chronicity (length of time) did not result in significant elevations of EBC IsoF. In fact, the individual who had been on oxygen the longest ( 20 years) reported the lowest value of IsoF in exhaled breath at $13 \mathrm{pg} / \mathrm{mL}$; the subject reporting oxygen use for the shortest period of time ( 3 weeks) generated the highest EBC IsoF level at $181 \mathrm{pg} / \mathrm{mL}$.

As it relates to oxygen concentration, relevance may only exist at higher concentrations/doses. There are many studies implicating high concentrations ( $\geq 40 \%$ ) of supplemental oxygen exposure as instigators of oxidative stress, however, there are no studies evaluating low-flow oxygen therapy as a mechanism of oxidative stress. The flows of oxygen therapy reported in this study were 2-4 $\mathrm{L} / \mathrm{min}$ (ie, $28-36 \%$ concentration). The lowest reported concentration of oxygen therapy in our study was $2 \mathrm{~L} / \mathrm{min}$, and this generated both the lowest $(11 \mathrm{pg} / \mathrm{mL}$ after lowflow domiciliary oxygen for $12 \mathrm{~h} / \mathrm{d}$ for 3 years) and the highest IsoF values $(181 \mathrm{pg} / \mathrm{mL}$ with low-flow domiciliary oxygen for $24 \mathrm{~h} / \mathrm{d}$ for 3 weeks). The subject who reported $4 \mathrm{~L} / \mathrm{min}$ of daily oxygen use for $9 \mathrm{~h} / \mathrm{d}$ for 5 years generated an EBC IsoF of $77 \mathrm{pg} / \mathrm{mL}$. The disparity in the range in IsoF compared to concentration alone suggests low-flow domiciliary oxygen does not influence oxidative stress. A $t$ test was performed to compare group EBC IsoF means. No statistical difference in mean IsoF levels was determined between the active control and active treatment groups $(P=.057)$. As a result of this finding, no changes to current low-flow domiciliary oxygen prescription practices are substantiated.

There were several limitations to this study. First, the study was likely underpowered. Little is understood currently about IsoF with the exception that it is a biosynthetic relative of the IsoPs, whose synthesis excels in the presence of supraphysiologic oxygen tension. We made the assumption that IsoF formation was predictable based on the research performed using IsoP as an oxidative stress biomarker, and this is reflected in the power analysis. The reality, however, is that there is a large gap in the literature regarding IsoF to make such assertions.

The lack of a standardized protocol for EBC collection introduces challenges in consistently and accurately interpreting and comparing results. Due to the inherent nature of the individual biomarker compounds being assessed, the various storage practices and specimen assessment tools (eg, assays vs mass spectrometry) make establishing a singular methodology for EBC collection and biomarker detection challenging. As a result, the American Thoracic Society/European Respiratory Society have only developed recommendations for specimen collection and storage.

Another limitation is that baseline levels of exhaledbreath IsoF in individuals diagnosed with COPD have yet to be defined. This makes the inability to fully interpret the obtained results a crucial limitation. It is understood that baseline levels exist in healthy individuals, but baseline levels of IsoF in exhaled breath have not been established. While this is considered a significant limitation of this study, our results will aid in establishing that benchmark knowledge for future studies. Ongoing research is needed to establish IsoF benchmark levels.

Elevated oxygen tension levels within the body can occur from both endogenous and exogenous sources. This study aimed to discern whether oxidative stress was occurring as a result of prescribed low-flow domiciliary oxygen therapy, an exogenous source of reactive oxygen species production. Endogenous sources of molecular oxygen can result from genetic or acquired mitochondrial diseases, such as amyotrophic lateral sclerosis or Huntington, Alzheimer, Lewy body, and Parkinson diseases. ${ }^{16}$ Study candidates were surveyed for these particular diseases, with none reported or observed. However, other more common diseases that are linked to mitochondrial dysfunction, such as diabetes and hypertension, were not controlled for because they were prevalent in this patient population.

Acquired mitochondrial dysfunction occurs as a result of adverse effects from drugs, infections, or other environmental factors. ${ }^{17}$ Diseases of the mitochondria appear to cause the most damage to cells of the brain, heart, liver, skeletal muscles, kidney, and the endocrine and respiratory systems. The goal of this study was to gain insight into the potential effect of oxygen to safely prescribe and manage oxygen therapy. ${ }^{17}$ The most common comorbidities reported by the participants in this study were COPD (100\%), hypertension (87\%), diabetes $(40 \%)$, coronary artery disease $(56 \%)$, and hyperlipidemia $(56 \%)$. Oxidative stress is central to many of these disease processes, with acquired mitochondrial dysfunction as a contributing factor. It is unknown currently to what degree, if any, these disease processes contribute to elevated IsoF levels as a result of liberated molecular oxygen from disrupted mitochondria. The limited understanding of contributing sources of elevated tissue oxygen tensions makes it challenging to interpret which source, endogenous or exogenous, is responsible for IsoF synthesis in this study, and to what extent. 


\section{Low-Flow Oxygen and OxidATive StREsS}

\section{Conclusions}

Oxygen is the most widely used and poorly regulated drug in the health care industry. ${ }^{18}$ While oxygen therapy provides clear benefits, its use is not without risk. Unregulated, liberal use of hyperoxic therapy can lead to oxygen toxicity and its adverse effects, such as oxidative stress. The ability to define the margin of safety between the effective dose and the toxic dose of oxygen would aid in mitigating insult and facilitate best practice outcomes. While the nonsignificant results of this study do not warrant changes to current oxygen-prescription practices, the resultant group differences were substantial enough to merit consideration that a trend was possible and, therefore, deserving of additional study. A larger recruitment of participants and tighter control of confounding comorbidities would provide a better platform from which to discern whether time and concentration of domiciliary oxygen therapy instigates ongoing oxidative stress.

\section{REFERENCES}

1. McGuiness AJA, Sapey E. Oxidative stress in COPD: sources, markers, and potential mechanisms. J Clin Med 2017;6(2):21.

2. Mach W, Thimmesch A, Pierce J, Pierce J. Consequences of hyperoxia and the toxicity of oxygen in the lung. Nurs Res Pract 2011; 2011:260482.

3. Birben E, Sahiner UM, Sackensen C, Erzurum S, Kalayci O. Oxidative stress and antioxidant defense. World Allergy Organ J 2012; 5(1):9-19.

4. Rahman I. The role of oxidative stress in the pathogenesis of COPD: implications for therapy. Treat Respir Med 2005;4(3):175-200.

5. Hedley-Whyte J, Sheridan DS. Pulmonary oxygen toxicity: investigation and mentoring. Ulster Med J 2008;77(1):39-42.

6. Morrow JD, Hill RF, Nammour TM, Badr KF, Roberts II LJ. A series of prostaglandin F2-like compounds are produced in vivo in humans by a non-cyclooxygenase, free radical-catalyzed mechanism. Proc Natl Acad Sci USA 1990;87(23):9383-9387.

7. Frijhoff J, Winyard PG, Zarkovic N, Davies SS, Stocker R, Cheng D, et al. Clinical relevance of biomarkers of oxidative stress. Antioxid Redox Signal 2015;23(14):1144-1170.

8. Fessel JP, Porter NA, Moore KP, Sheller KP, Roberts II LJ. Discovery of lipid peroxidation products formed in vivo with a substituted tetrahydrofuran ring (isofurans) that are favored by increased oxygen tension. Proc Natl Acad Sci USA 2002;99(26):16713-16718.

9. Continuous or nocturnal oxygen therapy in hypoxemic chronic obstructive lung disease: a clinical trial. Nocturnal Oxygen Therapy Trial Group. Ann Intern Med 1980;93:391-398.

10. Long term domiciliary oxygen therapy in chronic hypoxic cor pulmonale complicating chronic bronchitis and emphysema. Report of the Medical Research Council Working Party. Lancet 1981;1:681685 .

11. Deneke SM, Fanburg BL. Normobaric oxygen toxicity of the lung. 1980. N Engl J Med 1980;303(2):76-86.

12. Nagatomo F, Fujino H, Kondo H, Ishihara A. Oxygen concentration dependent oxidative stress levels in rats. Oxid Med Cell Longev 2012;2012:381763.

13. Vacchiano CA Osborne GR, Tempel GE. 8-ISO-PGF2alpha production by alveolar macrophages exposed to hyperoxia. Shock 1997 ; 9(4)266-273

14. Phillips M, Cataneo RN, Greenberg J. Effect of oxygen on breath markers of oxidative stress. Eur Respir J 2003;21:48-51.

15. Carpagnano GE, Kharitonov SA, Foschino-Barbaro MP, Resta O, Gramiccioni E, Barnes PJ. Supplementary oxygen in healthy subjects and those with COPD increases oxidative stress and airway inflammation. Thorax 2004;59(12):1016-1019.

16. Fessel JP, Hulette C, Powell S, Roberts II LJ, Zhang J. Isofurans, but not F2-isoprostanes, are increased in the substantia nigra of patients with Parkinson's disease and with dementia with Lewy body disease. J Neurochem 2003;85(3):645-650.

17. Ellinas H, Frost E. Mitochondrial disorders: a review of anesthetic considerations. Middle East J Anesthes 2011;21(2)235-244.

18. Bitterman H. Bench-to-bedside review: oxygen as a drug. Crit Care 2009;13(1):205. 